\title{
Piezopolymeric Transducer for Ultrasonic Sensorial Systems
}

\author{
A. Jiménez, A. Hernández, J. Ureña, J.J. García, J.M. \\ Villadangos, M. Mazo, C. De Marziani, M.C. Pérez \\ Electronics Department \\ University of Alcalá \\ Alcalá de Henares (Madrid), Spain \\ Email: ajimenez@depeca.uah.es
}

\author{
F.J. Álvarez \\ Electronics Department \\ University of Extremadura \\ Cáceres, Spain \\ Email: fernando@depeca.uah.es
}

\begin{abstract}
This paper presents a prototype of an ultrasonic transducer based on electromechanical film (EMFi). This electromechanical film provides a wide bandwidth response, useful for robotic applications. Furthermore, its sensitivity is constant enough to allow echo-pulse measurement in the desired frequency range. The emission patterns are obtained for different configurations, verifying the correspondence between experimental data and theoretical piston-like model. Results suggest that the polymer can be suitably used as a broadband ultrasonic transducer, in order to improve performances in ultrasonic sensorial systems, where the emission encoding with binary sequences, and the used modulation schemes, require considerably large bandwidth.
\end{abstract}

\section{INTRODUCTION}

Recently an increasing interest about ultrasonic transducers has appeared, due to the wide range of different applications. The most used ones have been the piezoelectric and electrostatic transducers, though it is necessary to improve their sensibility, bandwidth and/or size. The advancement in material research has given new options based on electrect materials and composites, or miniature electret microphones integrated on silicon chips, as microelectro-mechanical systems (MEMS). Indeed, products using electrets have been considered in many applications, being widely used in acoustic microphones, or even suggested for biomedical applications [1] [2].

In the field of robot navigation, broadband air ultrasonic transducers are of great importance. They should operate in a large frequency range $(20 \mathrm{kHz}-200 \mathrm{kHz})$ with high sensibility. Unfortunately, these requirements can not be often achieved by commercially available airborne ultrasonic transducers. Hence, new transducer technologies are required to develop broadband ultrasonic transducers with good adaptation to air. The interest in these features comes from the fact that ultrasonic signal is often encoded and modulated. In this way, broadband transducers imply less constraint in algorithms proposed for ultrasonic signal processing, allowing also the obtaining of additional spectral parameters from environment and reflectors. One promising transducer material for that is a cellular quasi-polymer electrect film, called electro mechanical film (EMFi) [3].
One kind of piezo-polymeric electret [4] is poly-vinylidene fluoride (PVDF), which is widely used in ultrasonic transducers [1] [5]. Other option is cellular piezoelectric polymer, which became an important research item in the late 1990 's [1] [4], when properly charged cellular polypropylene (PP) was shown to possess longitudinal piezoelectric coefficient about 10 times higher than those from PVDF [2] [6]. More recently, micro-porous polypropylene (PP) foams, defined as electromechanical films (EMFi), were introduced [7] and further developed in Finland by VTT and several small companies [8]. In last years significant progress has been carried out in this field [4] [5], and many applications have been suggested related to electromechanical, electroacoustic, underwater, and ultrasonic sensors and actuators [5].

This work describes the use of EMFi piezo-polymer material to design air-borne ultrasound transducers in a versatile way. The obtained acoustic features are detailed, as well as some basic configurations. The paper is organized as follows: Section II presents the transducer manufacturing, firstly describing the used material, and following with the design and manufacturing of a prototype; Section III is dedicated to the ultrasonic transducer characterization, with simulation and experimental results from an ad-hoc driving system; and, finally, some conclusions are discussed in Section IV.

\section{TRANSDUCER FABRICATION}

\section{A. Piezopolimeric material}

Piezoelectricity refers to changes in the internal polarization of a dielectric material for small changes in stress and vice versa. A material can be piezoelectric because of its crystalline symmetry, like traditional polar piezopolymers. There exist also several polymers where piezoelectricity can be induced by external electric field, which permanently aligns electric quasidipoles in the material, as cellular space-charge electrets [9]. Therefore in this case the symmetry is broken in a macroscopic level. Physical characterization reveals close analogies between cellular polymers and ferroelectric materials, so the material can be considered also as ferroelectret [9]. 
Electromechanical film (EMFi) is a thin porous polypropylene film with biaxially oriented flat voids, as lentils, with a lateral dimension of $10-100 \mu \mathrm{m}$ and a vertical one between 1 and $5 \mu \mathrm{m}$ [9], as shown in Fig. 1a. It is obtained through extrusion, biaxial stretching, and controlled inflation by pressure patented treatments [7]. This heterogeneous foam film exhibits a permanent space-charge after corona or electrode charging with high electric fields during the manufacturing process. It has been tested that the charge distribution in cellular foams is such that the upper surfaces of all gas voids are charged to one polarity, and the lower surfaces to the opposite one, as quasi-dipoles (Fig. 1b), due to micro discharges in voids [10]. More detailed description of the process can be found in [7] [11].

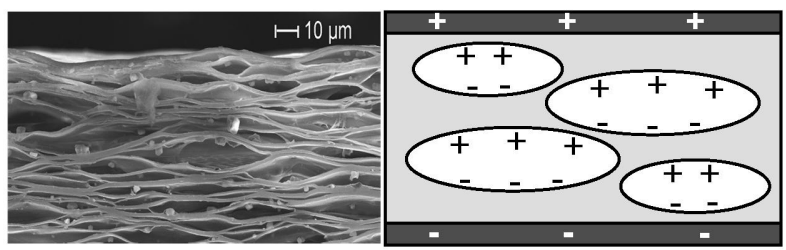

Fig. 1. Cross-section image of cellular polypropylene [9] (a). Charged voids in the foam, responsible for the quasi-piezoelectricity, represent the structure and the inhomogeneous charge distribution (b).

Finally electrodes are evaporated or glutted. From the application point of view, it is important to take into account that if the metal coat is too rigid, the quasi-piezoelectric response of the film (a charge) will be small, whereas the output impedance of the sensor will be too high. Moreover, mechanical clamping will shift the quasi-piezoelectric resonance of the transducer towards high frequencies [12].

As shown in Fig. 1b, the charged voids in cellular polymers form perfectly-oriented macroscopic quasi-dipoles. Due to the extremely large anisotropy of the investigated systems, longitudinal piezoelectric constant (d33) is the dominant quasipiezoelectric coefficient [13]. Dielectric and interferometric measurements allow to determine the resonance frequencies of the films, which are in the range of a few hundreds of $\mathrm{kHz}$ [12] [14], as well as the frequency response of the d33-coefficient, minimum just below the thickness resonance [14]. In cellular polymers, $\mathrm{d} 33$ is positive (intrinsic quasi-piezoelectricity); $\mathrm{d} 31$ and $\mathrm{d} 32$ take low negative values and low piroelectric coefficients. Therefore, the quasi-piezoelectric coefficients in piezoelectric-like foams, as EMFi, are opposite to those in ferroelectric polymers, as PVDF; and they have the same sign as those in typical ferroelectric ceramics, as lead zirconate titanate (PZT). Moreover, large values, up to several hundred $\mathrm{pC} / \mathrm{N}(130-450 \mathrm{pC} / \mathrm{N})$, are commonly achieved for $\mathrm{d} 33$, which is more than one order of magnitude larger than in conventional ferroelectric polymers (20-30pC/N for PVDF) [6] [8] [12]. This is mainly attributed to the very low Young's modulus (about 2MPa), compared to other common piezoelectrics, e.g., PVDF and PZT [15] [16]. The acoustic impedance is only $2.6 \times 10^{4} \mathrm{~kg} /\left(\mathrm{m}^{2} \mathrm{~s}\right)$, providing a suitable matching to air [17].

When exposed to dynamic mechanical or acoustic energy, the structure generates an electric charge, operating as a receiver. The effect also acts in reverse, converting electrical energy into vibration and sound, behaving as an actuator. This dual operation opens the scope of applications. Considering an EMFi-based transducer working in thickness mode, when an external force is applied, the thickness of the film changes. This variation depends on the kind of layer in the structure, due to the different elastic behavior of the polymer and to voids (intrinsic quasi-piezoelectricity). As a consequence, a mirror charge proportional to the force is induced at electrodes. This charge can be measured with a charge amplifier, or with a voltage amplifier with very high input impedance.

Sensitivity of EMFi films is strongly dependent on the thermal environment during processing, storage, and operation time. Thus, the charge tends to decrease if temperature remains over $50^{\circ} \mathrm{C}$ for a long period of time [17].

In summary, ferroelectrets have some advantages compared to conventional piezoelectric materials based on ceramics or polar polymers. Apart from their high d33-coefficients, they are thin and lightweight, and they may be obtained in almost any size and/or shape. They can be easily handled and they are inexpensive. Their relatively small specific acoustic impedance is an advantage for many transducer applications. The main drawback is the reduced stability of the piezoelectric effect at temperatures over $50^{\circ} \mathrm{C}$. This feature of the material can limit its application range, although related improvements are currently under research.

\section{B. Transducer prototype}

The developed transducer based on EMFi films is depicted in Fig. 2a. For experimental research, EMFi electret foil with a nominal thickness of $70 \mu \mathrm{m}$, metallic on one side with aluminium electrodes, has been used. The film has been cut in different shapes (square and round) and sizes, and mounted on a pad, so the piezo-material can oscillate in thickness mode. The polymer has been fixed on one side using conductive adhesive (Ag paste), acting as the second electrode. In Fig. 2b a prototype with an active area of $1.5 \times 1.3 \mathrm{~cm}^{2}$ is shown.
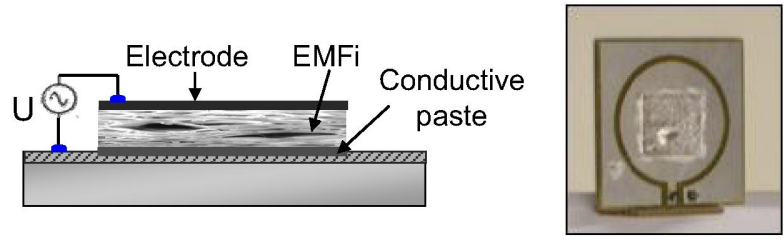

Fig. 2. Cross-section diagram of transducer (a). Prototype transducer (b). 


\section{ULTRASONIC ACTUATOR CHARACTERIZATION}

Different EMFi-based (ferroelectret) emitters have been assembled and their sound output and directivity measured. For that, it has been necessary to develop a driving system to suitably excite the transducer up to $600 \mathrm{~V}_{\mathrm{pp}}$.

\section{A. Driving system}

The response of the EMFi-based emitter is proportional to the used bias voltage. In fact, it has been shown that the deflection of the polymer has a strong dependence on the applied driving voltage [3]. Higher Sound Pressure Levels (SPL) are therefore achieved at higher driving voltages, being desirable to excite with a voltage amplitude higher than $300 \mathrm{~V}_{\mathrm{pp}}$ [3]. Nevertheless, as a result of discharges occurring at the boundary of the polymer, the maximum SPL is limited at a maximum driving voltage around $1000 \mathrm{~V}_{\mathrm{pp}}$. The displacement of the polymer surface is relatively constant in the frequency range between $20 \mathrm{kHz}$ and $200 \mathrm{kHz}$ [3]. These both considerations define the work conditions and, therefore, the driver system specifications.

The global emission stage has a first control module that provides the digital codification of the ultrasonic signal $v_{e}(t)$ to be emitted. This signal $v_{e}(t)$ is generated by a low-cost FPGA device [18], together with a DA converter. Fig. 3 shows the block diagram of the circuit developed to suitably excite the transducer, starting from the signal $v_{e}(t)$.

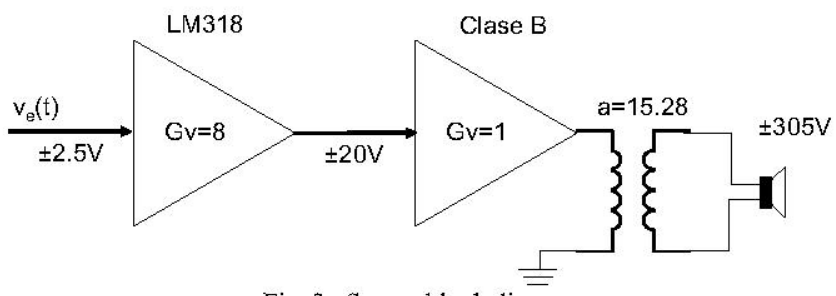

Fig. 3. Sensor block diagram.

As mentioned before, this driving system should consider constraints given by the EMFi-based emitter, regarding the excitation voltage (reaching the $300-600 \mathrm{~V}_{\mathrm{pp}}$ range), the bandwidth $(20 \mathrm{kHz}-200 \mathrm{kHz})$ and the equivalent capacitance (14 $\mathrm{pF} / \mathrm{cm}^{2}$ in this specific case [8]). The stage consists of a voltage amplifier, a class B amplifier and a transform set, which provides a maximum value of approximately $600 \mathrm{~V}$. The first stage of amplification, with a voltage gain of 8 , is a noninverter configuration, based on LM318 operational amplifier [19], with enough bandwidth and slew-rate for the application. In next block, the signal goes through a class-B amplifier, with complementary bipolar transistors, and unit voltage gain. Finally, the amplified output is connected to the transformer stage, with a ratio of 15.28 between primary and secondary.

\section{B. Characterization results and discussion}

The acoustical characterization has been performed in a compact acoustic chamber, covered with highly-sound- absorbing polymer foam, in order to avoid significant reflections of sound waves from the chamber walls. A calibrated Brüel and Kjaer eighth-inch (4138) pressure-field microphone [20] has been employed for the characterization. It was placed at $25 \mathrm{~cm}$ on the axial axis from the EMFi-based emitter (through-transmission mode). The sound level, emitted by an EMFi transducer with an active area of $2 \times 2 \mathrm{~cm}^{2}$, has been measured in a wide frequency range $(20 \mathrm{kHz}-140 \mathrm{kHz})$, with $300 \mathrm{~V}_{\mathrm{pp}}$ sinusoidal voltage. The frequency response is shown in Fig. 5, providing a stable response for the mentioned range.

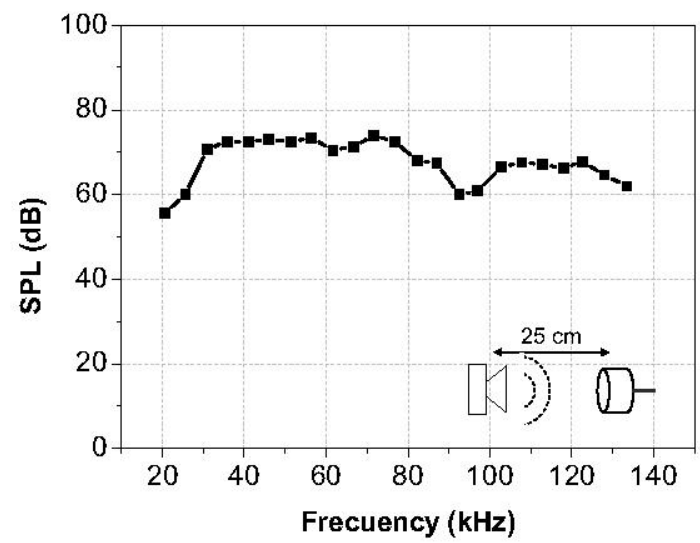

Fig. 5. Sound Pressure Level (SPL) of the EMFi-based emitter at different frequencies.

The directional properties of the EMFi actuator at different frequencies $(30 \mathrm{kHz}, 60 \mathrm{kHz}, 80 \mathrm{kHz}$ and $110 \mathrm{kHz})$ are presented in Fig. 6. The emission patterns show how the main lobe becomes narrower when the frequency increases. The amplitudes of the sidelobes are smaller compared to the main one; nevertheless, the number of them increases with frequency. This trend is similar to the one shown by pistonbased model in an infinite rigid baffle.
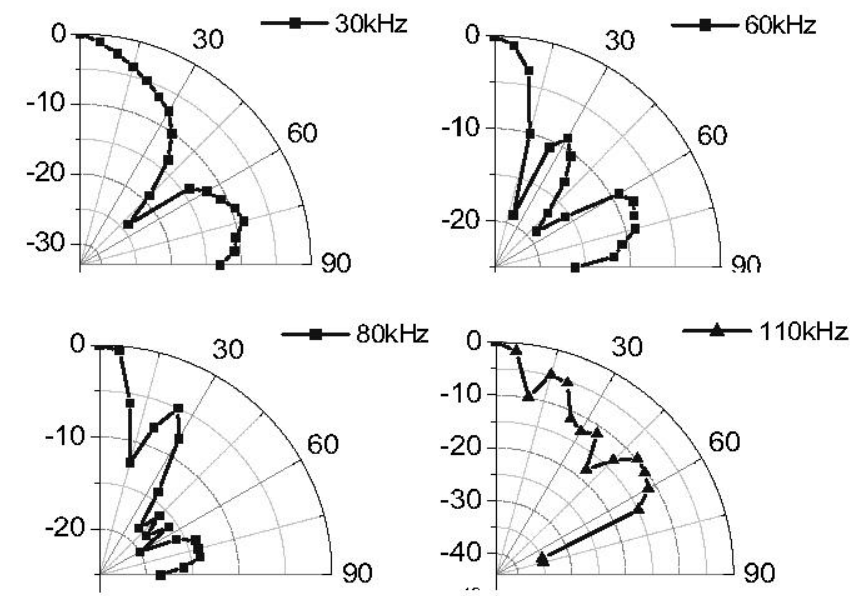

Fig. 6. Emission patterns of the EMFi-based emitter at $30 \mathrm{kHz}, 60 \mathrm{kHz}, 80 \mathrm{kHz}$ and $110 \mathrm{kHz}$, with $300 \mathrm{Vpp}$ at $25 \mathrm{~cm}$.

According to the radiation patterns in Fig. 6, EMFi-based emitter becomes more directive with frequency: the total beam 
angle decreases $(-6 \mathrm{~dB})$ when increasing the frequency. Total beam angle is around $50^{\circ}$ at $30 \mathrm{kHz}$, decreasing until $30^{\circ}$ when frequency is $60 \mathrm{kHz}$; analogously, at $80 \mathrm{kHz}$ the total beam angle is around $20^{\circ}$ whereas it is around $15^{\circ}$ at $110 \mathrm{kHz}$.

\section{Simulation response}

The EMFi material can not be described with a piezoelectric material model, mainly due to its inhomogeneous structure, so a more complex microscopic model should be developed. Nevertheless, from a macroscopic point of view, and as a first approximation, the EMFi-based transducer can be considered as a piston source.

The radiation field produced at the polar point $(r, \theta)$ by a free piston-type of radius $a$ in far field, assuming it is located in an infinite baffle, can be represented by [21]:

$$
p(r, \theta, t)=j P(r) \cdot e^{j(w t-k r)} H(\theta)
$$

Where:

$$
\begin{aligned}
& P(r)=\frac{\rho_{0} \cdot c}{2} U_{0} \frac{a}{r} k a \\
& H(\theta)=\left|\frac{2 J_{1}(k a \cdot \sin \theta)}{k a \cdot \sin \theta}\right|
\end{aligned}
$$

Where $k$ is the wave number; $c$ is the speed of sound; $J_{1}$ is the first order Bessel function; $\mathrm{U}_{0}$ the initial amplitude; and $\rho_{0}$ is the volumetric air density.

The emission patterns, obtained according to (1) at different frequencies $(30 \mathrm{kHz}, 60 \mathrm{kHz}, 80 \mathrm{kHz}$ and $110 \mathrm{kHz})$, are shown in Fig. 7. It is possible to observe how, for a given distance from the source, when frequency increases (i.e., $k \cdot a=2 \pi \cdot a / \lambda$ increases), the main lobe width decreases whereas the number of side lobes is higher, in a similar way to the experimental pattern (see Fig. 6)
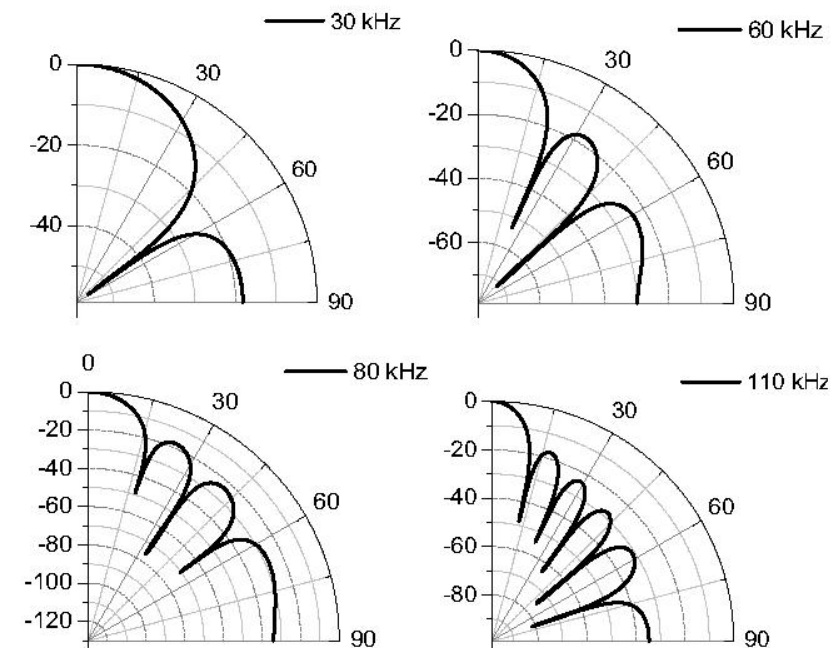

Fig. 7. Simulated piston emission patterns at $30,60,80$ and $110 \mathrm{kHz}$, according to (1).

Comparing radiation patterns in Fig. 6 and 7, the number of sidelobes is significantly higher in the piston model, but also narrower at higher frequencies, being similar at lower ones. The discrepancies can be related to two main factors: firstly, the low spatial resolution in the experimental patterns $\left(5^{\circ}\right)$, that makes impossible to distinguish very narrow sidelobes, as the ones observed in the theoretical pattern at high frequencies. Secondly, small differences are likely, since the transducer is a film working in thickness mode glutted at a back electrode. This implies a boundary effect, which is not adequately predicted by the piston in an infinite baffle model.

On the other hand, considering the total beam angle, theoretical and experimental results give similar values at different frequencies, confirming the piston-like behavior of the EMFi actuator.

\section{APPLICATION EXAMPLE}

Some experimental tests have been carried out to compare the EMFi actuator with a commercial one to confirm the influence of the bandwidth. The reference used has been one of the most popular transducer in robotic applications, the SensComp's serie 600 [22], which operates at its resonance frequency of $50 \mathrm{kHz}$ with $12 \mathrm{KHz}$ bandwidth. Previous works in literature have used ultrasonic signal encoding to develop advanced sensors (with several emitters and/or receivers), by using pseudo-random sequences [23] [24], Barker codes [25], or Golay codes [26]. In this case, and as an example, a 255-bit Kasami code has been used, which is modulated at $50 \mathrm{kHz}$ [27].

The direct ultrasonic emission is recorded by a condenser microphone capsule Avisoft Bioacoustics CM16 [29] placed at 30 inches. The acquired signal is demodulated and correlated with the corresponding 255-bit Kasami code to determine the arrival of the transmission. Fig. 8 shows the correlated result for the ideal case. Note that the auto-correlation function provides a main peak, which depends on the length of the sequence (255 bits) and on the modulation parameters (oversampling and number of carrier periods per transmitted bit).

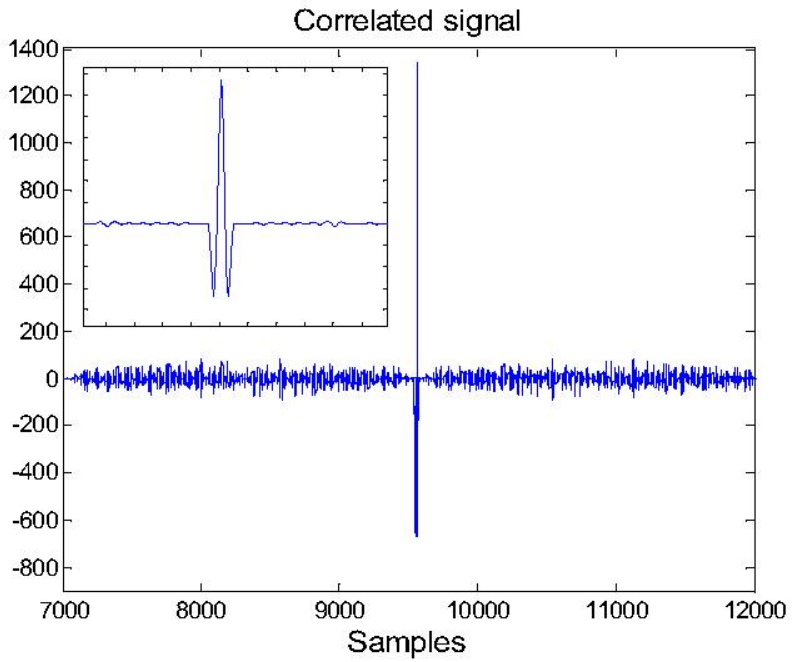

Fig. 8. Correlation peak for the ideal case. 
The received signals are shown in Fig. 9 and 10, with the ones obtained after correlation for both transducers, respectively. The first observed effect, due to bandwidth limitations, is the appearance of sidelobes around the peak of the correlation process. The main peak obtained with the EMFi actuator is around $20 \%$ higher than the reference, what implies a remarkable improvement. Furthermore, the main advantage to point out is the ratio between the main peak and the sidelobes, which is significantly higher for the EMFi's one. In fact, the ratio for the reference transducer is $20 \%$, whereas for EMFi it is $73 \%$. This implies an increasing in the signal-tonoise ratio (SNR) supported by the system, therefore a higher accuracy and noise immunity.
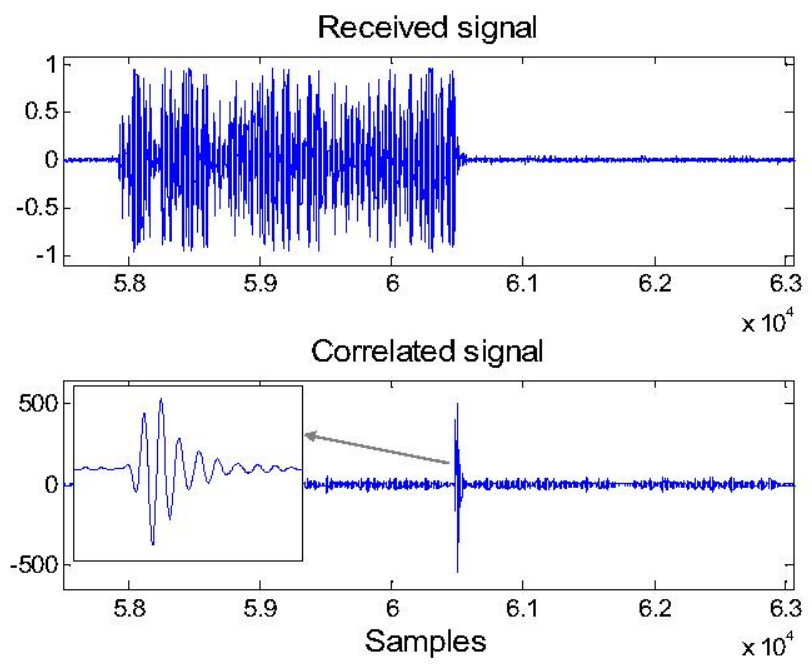

Fig. 9. Received signal and correlation results for the reference SensComp transducer.
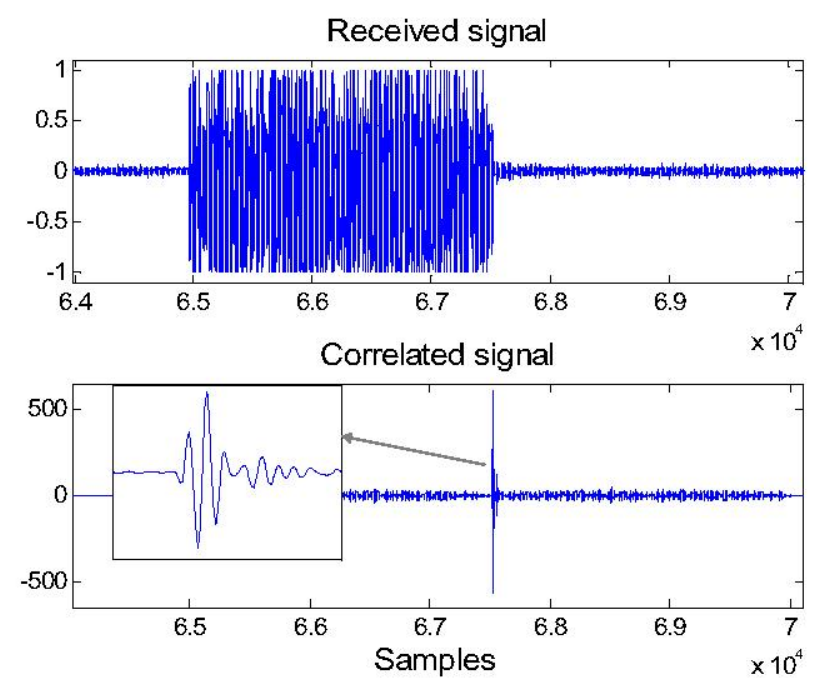

Fig. 10. Received signal and correlation results for the EMFi-based transducer

\section{CONCLUSIONS}

The proposal of an EMFi-based ultrasonic transducer has been described and the manufacturing of first prototypes has been illustrated. It has been shown that EMFi-based sensors offer high potential performances for robotic applications, in terms of efficiency and frequency range, as well as low manufacturing costs. A conditioning system has been proposed to drive the EMFi-based emitter between $300 \mathrm{~V}_{\mathrm{pp}}$ and $500 \mathrm{~V}_{\mathrm{pp}}$ in a wide frequency range $(20 \mathrm{kHz}-200 \mathrm{kHz})$. The frequency response emitted by the EMFi-based transducer shows a stable behaviour for all the considered range. The measured emission pattern at different frequencies is in suitable correspondence with the theoretical behaviour of a piston source in an infinite baffle. Finally, an experimental test has confirmed the promised behaviour of the EMFi ultrasonic actuator in robotic applications.

\section{ACKNOWLEDGMENT}

This work has been possible by funding from the Madrid Community (INCUBUS project CAM-UAH2006), the Spanish Ministry of Education and Science (RESELAI project TIN2006-14896-C02-01) and from the IMSERSO (ARIADNA project 212/05).

\section{REFERENCES}

[1] E. Fukada "History and Recent Progress in Piezoelectric Polymers", IEEE Transactions on ultrasonics, ferroelectrics, and frequency control, vol. $47, n^{\circ} 6$, pp. $1277-1290,2000$

[2] J. Lekkala, R. Poramo, K. Nyholm, and T. Kaikkonen, "EMF force sensor - a flexible and sensitive electret film for physiological applications," Med. and Biolog. Engin..\& Comput. vol. 34, pp. 67-68, 1996.

[3] Alexander Streicher, Rolf Müller, Herbert Peremans, Reinhard Lerch, "Broadban Ultrasonic transducer for a Artificial Bat Head", IEEE ultrasonics Symposium, pp. 1364-1367, 2003.

[4] R. Gerhard-Multhaupt, "Less can be more - holes in polymers lead to a new paradigm of piezoelectric materials for electret transducers," IEEE Trans Diel. Electric. Insulat., vol. 9, pp. 850-859, 2002.

[5] S. Bauer, R. Gerhard-Multhaupt, and G. M. Sessler, "Ferroelectrets: Soft electroactive foams for transducers," Physics Today, vol. 57, No. 2, pp. $37-43,2004$.

[6] G. M. Sessler and J. Hillenbrand, "Electromechanical response of cellular electret films," Appl. Phys. Letters, vol. 75, pp. 3405-3407, 1999.

[7] K. Kirjavainen, "Electromechanical film and procedure for manufacturing same", U.S. Patent No. 4654 546, 1987.

[8] M. Paajanen, J. Lekkala, and K. Kirjavainen, "ElectroMechanical Film EMFi._A New Multipurpose Electret Material", Sensors and Actuators A, Vol. 84, pp. 95_102, 2000.

[9] M. Lindner, H. Hoislbauer, R. Schwödiauer, S. Bauer-Gogonea and S. Bauer "Charged Cellular Polymerswith"Ferroelectretic'Behavio".. IEEE Transactions on Dielectrics and Electrical Insulation Vol. 11, No. 2; April 2004, pp.255-263

[10] M. Lindner, S. Bauer-Gogonea, S. Bauer, M. Paajanen, and J. Raukola, "Dielectric barrier microdischarges: Mechanism for thr charging of cellular piezoelectric polymers," J. Appl. Phys., vol. 91, pp. 5283-5287, 2002.

[11] A. Savolainen, K. Kirjavainen, Electrothermomechanical film: Part 1. Design and characteristics, J. Macromol. Sci. -Chem. A26_2-3. $1989.583-591$.

[12] G. S. Neugschwandtner, R. Schwodiauer, M. Vieytes, S. BauerGogonea, S. Bauer, J. Hillenbrand, R. Kressmann, G. M. Sessler, M. Paajanen, and J. Lekkala, "Large and broadband piezoelectricity in smart 
polymer-foam space-charge electrets", Appl. Phys. Letters, vol. 77, pp. $3827-3829,2000$

[13] G. S. Neugschwandtner, R. Schw"odiauer, S. Bauer-Gogonea, S. Bauer, M. Paajanen, and J. Lekkala, "Piezo- and Pyroelectricity of a Polymerfoam Space-charge Electret”, J. Appl. Phys., Vol. 89, pp. 4503_4511, 2001.

[14] J. Hillenbrand and G. M. Sessler, "Quasistatic and dynamic piezoelectric coefficients of polymer foams and polymer film systems", IEEE Trans. Dielectrics and Electrical Insulation, vol. 11, pp. 72-79, 2004.

[15] X. Zhang, J. Hillenbrand, and G. M. Sessler, "Improvement of piezoelectric activity of cellular polymers using a double-expansion process", J. Phys. D: Appl. Phys., vol. 37, pp. 2146-2150, 2004.

[16] M. Wegener, W. Wirges, R. Gerhard-Multhaupt, M. Dansachmtiller, R. Schwddiauer, S. Bauer-Gogonea, S. Bauer, M. Paajanen, H. Minkkinen, and J. Raukola, "Controlled inflation of voids in cellular polymer ferroelectrets: Optimizing electromechanical transducer properties," Appl. Phys. Lett., vol. 84, pp. 392-394, 2004.

[17] http://www.emfit.com/products

[18] A. Hernández, J. Ureña, J. J. García, M. Mazo, J. P. Dérutin, J. Serot, "Estudio comparativo de arquitecturas para el tratamiento en tiempo real de la información procedente de transductores ultrasónicos con funcionamiento multi-modo", Actas del Seminario Anual de Automática, Electrónica Industrial e Instrumentación (SAAEI'02), vol. 2, pp. 201204, Alcalá de Henares, 2002

[19] National Semiconductor, "LM118/LM218/LM318 Operational Amplifiers", Datasheet Document, 2000
[20] Brüel and Kaejer: www. bksv.com

[21] L. E. Kinsler et al. "Fundamentos de acústica" Mexico, Ed. LIMUSA, 1999

[22] SensComp's Series 600, Product Spec. www.senscomp.com

[23] K. W. Jörg and M. Berg. Using Pseudo-random Codes for Mobile Robot Sonar Sensing. In Proc. of th 3rd IFAC Symposium on Intelligent and Autonomous Vehicles (LAV'98), pp. 231-236, 1998.

[24] A. Heale and L. Kleeman. A Sonar Sensing with Random Double Pulse Coding. In Proc. of the Australian Conference on Robotics and Automation, pp 81-86, 2000.

[25] H. Peremans, K. Audenaert, and J. Van Campenhout. A high-resolution sensor based on tri-aural perception. IEEE Trans. on Robotics and Automation, vol. 9(1), pp. 36-48, 1993.

[26] A. Hernández, J. Ureña, J. J. García, V. Díaz, M. Mazo, D. Hernanz, J. P. Dérutin and J. Serot. Ultrasonic signal processing using configurable computing. In Proc. of the 15th Triennial World Congress of the International Federation of Automatic Control (IFAC'02), 2002.

[27] T. Kasami. Weight distribution formula for some class of cyclic codes. Combinational Mathematics and its Applications. University of North Carolina Press, Chapel Hill, N.C., 1969.

[28] Avisoft Bioacoustics UltraSoundGate microphone capsule CM16; Product Spec., 2006. 